\title{
Morfologia da camada nitrocarbonetada a plasma do aço DIN $100 \mathrm{Cr} 6$
}

\section{Morphology of the plasma nitrocarburized layer on DIN $100 \mathrm{Cr} 6$ steel}

\author{
Marcos Alves Fontes ${ }^{1}$, Vladimir H. B. Scheid ${ }^{2}$, David S. Machado ${ }^{3}$, Luiz C. Casteletti ${ }^{3}$, Pedro A. P. Nascente ${ }^{1,4}$
}

\section{RESUMO}

Os tratamentos superficiais de ligas metálicas melhoraram as características das superfícies por meio da deposição de outro material ou por meio da modificação superficial, por exemplo, com a formação de revestimentos. A nitrocarbonetação, considerado um dos tratamentos termoquímicos existentes mais importantes, envolve a difusão simultânea dos elementos nitrogênio e carbono na superfície do componente. O entendimento e o controle da formação da camada nitrocarbonetada apresentam considerável interesse industrial, devido às melhorias alcançadas nas resistências ao desgaste, fadiga e à corrosão das ligas. Neste trabalho, foi empregado o processo de modificação superficial por nitrocarbonetação a plasma no aço DIN 100Cr6, variando-se a temperatura de tratamento $\left(550^{\circ} \mathrm{C}\right.$ e $\left.600{ }^{\circ} \mathrm{C}\right)$ e a concentração de $\mathrm{CH}_{4}$ na mistura $(0 \%, 1 \%, 1,5 \%$ e $2 \%)$, com tempo de tratamento fixo em 2 horas. As amostras foram caracterizadas por meios das técnicas de DRX, MEV e EDS. Os resultados mostram que a morfologia e a constituição da camada composta são bastante sensíveis à temperatura e à composição química da atmosfera de tratamento. O aumento na quantidade de carbono na mistura gasosa contribui para a formação da fase $\varepsilon$, e o aumento da temperatura promove maiores espessuras da camada composta, da zona de difusão e da camada de micro-porosidade formada.

Palavras-chave: Modificação superficial; Aço; Nitrocarbonetação; Metano; Micro-porosidade.

\begin{abstract}
The surface treatments of metallic alloys improve the surface characteristics by the deposition of another material or by surface modification, for instance, with the production of coatings. The nitrocarburizing, considered one of the most important thermochemical treatments, involves the simultaneous diffusion of nitrogen and carbon into the surface. Understanding and controlling the formation of the nitrocarburized layer have considerable industrial interest due to the improvements regarding wear, fatigue, and corrosion resistances of the alloys. In this work, it was performed a surface modification on DIN $100 \mathrm{Cr} 6$ steel by plasma nitrocarburizing process. The variables were the treatment temperature $\left(550^{\circ} \mathrm{C}\right.$ and $\left.600^{\circ} \mathrm{C}\right)$ and methane concentration in the gas mixture $(0 \%, 1.0 \%, 1.5 \%$, and $2.0 \%)$, while the treatment time was $2 \mathrm{~h}$. XRD, SEM, and EDS analyses were used to characterize the samples. The results show that the compound layer composition and morphology are quite sensitive to the temperature and chemical composition of the treatment atmosphere. The presence of carbon in the gas mixture contributes to the formation of the $\varepsilon$ phase, and elevated temperatures cause an increase in the thickness of the compound layer, diffusion zone, and micro-porosity layer.
\end{abstract}

Keywords: Surface modification; Steel; Nitrocarburizing; Methane; Micro-porosity.

\footnotetext{
1 Universidade Federal de São Carlos - Programa de Pós-Graduação em Ciência e Engenharia de Materiais - São Carlos (SP) - Brasil ${ }^{2}$ Departamento de Ciência e Tecnologia Aeroespacial - Instituto de Estudos Avançados - São José dos Campos (SP) - Brasil ${ }^{3}$ Universidade de São Paulo - Escola de Engenharia de São Carlos - Departamento de Engenharia de Materiais - São Carlos (SP) - Brasil ${ }^{4}$ Universidade Federal de São Carlos - Departamento de Engenharia de Materiais - São Carlos (SP) - Brasil Correspondence author: Marcos A. Fontes - Universidade Federal de São Carlos - Programa de Pós-Graduação em Ciência e Engenharia de Materiais Rod. Washington Luis, km 235 - São Carlos (SP) - Brasil - CEP:13565-905 - São Carlos (SP) - Brasil E-mail: fontesma@hotmail.com
}

Recebido: 31/08/2017 Aprovado: 22/11/2017 


\section{INTRODUÇÃO}

O tratamento termoquímico de nitrocarbonetação é o processo que introduz nitrogênio e carbono na superfície do material, com o objetivo de alterar as propriedades da mesma, dentre elas a resistência ao desgaste, resistência à fadiga, resistência à corrosão e dureza superficial. Essas propriedades são modificadas devido à formação de fases cristalinas na superfície das peças, chamadas de nitretos e/ou carbonitretos de ferro ${ }^{(1)}$, compostas por átomos de carbono, nitrogênio e ferro, onde há a ocorrência da difusão desses elementos na forma atômica ou combinada para o interior do material.

O processo de nitrocarbonetação pode ser realizado em temperaturas acima ou abaixo da temperatura de transformação eutetóide do diagrama Fe-N. A nitrocarbonetação ferrítica éo nome dado ao processo realizado na temperatura abaixo da temperatura de transformação eutetóide, e é caracterizada por apresentar uma camada superficial formada pela camada composta e pela zona de difusão, subjacente a primeira. No caso da nitrocarbonetação austenítica, realizada em temperaturas acima da temperatura de transformação eutetóide, ocorre a transformação parcial da matriz ferrítica em austenítica, e, portanto, além da formação da camada composta e da zona de difusão, há a formação de uma terceira camada, entre as duas citadas anteriormente, denominada de camada de austenita transformada. Esta camada de austenita pode se transformar em martensita durante um resfriamento rápido da amostra, elevando por consequência a dureza na região abaixo da camada composta ${ }^{(2)}$.

As estruturas formadas durante o processo de nitrocarbonetação têm apresentado propriedades bastantes atraentes do ponto de vista científico e tecnológico. A obtenção de propriedades como ótima resistência mecânica, grande estabilidade química e boas propriedades magnéticas motivou nos últimos anos intensas pesquisas nesta área dos tratamentos termoquímicos. Já existem atualmente inúmeras aplicações para estes materiais, principalmente ligadas às indústrias do setor metal-mecânico ${ }^{(3)}$, tais como a fabricação de motores automotivos mais leves e duráveis ${ }^{(4,5)}$ e a utilização de ferramentas mecânicas com maior resistência e maior durabilidade.

A utilização em larga escala e o controle das propriedades microestruturais dos nitretos e dos carbonitretos constituem atualmente grandes desafios para se tornar viável a utilização deste processo, trazendo como benefício grande valor estratégico do ponto de vista econômico e científico. Sendo assim, o objetivo deste trabalho é o entendimento de quais variáveis de entrada, se controladas, sejam capazes de formar uma camada homogênea endurecida de carbonitretos $\varepsilon-\mathrm{Fe}_{2-3}(\mathrm{C}, \mathrm{N})$, na superfície da liga metálica DIN 100Cr6, fazendo o uso da nitrocarbonetação com plasma pulsado e temperaturas relativamente baixas. A ideia é garantir o entendimento da relação entre alguns parâmetros de processo, como temperatura e atmosfera de tratamento, na formação das fases na superfície das amostras, e na definição da espessura das camadas superficiais formadas.

A liga DIN 100Cr6 escolhida para o estudo é utilizada como matéria-prima na fabricação de um componente mecânico aplicado em compressores herméticos para refrigeração.

\section{PROCEDIMENTOS EXPERIMENTAIS}

As amostras do aço DIN 100Cr6, cuja composição química está apresentada na tabela 1 , foram nitrocarbonetadas a plasma, utilizando uma descarga luminescente d.c. pulsada e uma pressão de trabalho de $3 \mathrm{mbar}$, em um equipamento industrial MP-250 da Metal Plasma Ltda (Fig. 1).

A tensão aplicada (na faixa de 300 a $500 \mathrm{~V}$ ) bem como a corrente utilizada, foram ajustadas de tal forma a atingir e garantir as temperaturas desejadas. Existem outras variáveis envolvidas, como por exemplo a temperatura e a área total das peças expostas ao plasma, que influenciam nos ajustes dos parâmetros de tensão e corrente.

Ainda, utilizou-se o tempo de nitrocarbonetação de $2 \mathrm{~h}$, temperaturas de $550 \pm 5{ }^{\circ} \mathrm{C}$ e $600 \pm 5{ }^{\circ} \mathrm{C}$ (temperaturas abaixo e acima da temperatura de transformação eutetóide de $590{ }^{\circ} \mathrm{C}$ ), medidas por meio de dois termopares fixos nas bandejas de apoio das peças, e atmosferas contendo $\mathrm{H}_{2}-74 \% \mathrm{~N}_{2}$ e concentrações de 1, 1,5 e 2,0\% de $\mathrm{CH}_{4}$ (concentrações relacionadas ao fluxo total de gás inserido na câmara, em $\mathrm{sccm}$ ). As amostras também foram nitretadas em uma atmosfera de $\mathrm{H}_{2}-75 \% \mathrm{~N}_{2}$.

$\mathrm{O}$ resfriamento das amostras ocorreu de forma lenta, dentro da câmara, com o uso de uma atmosfera inerte.
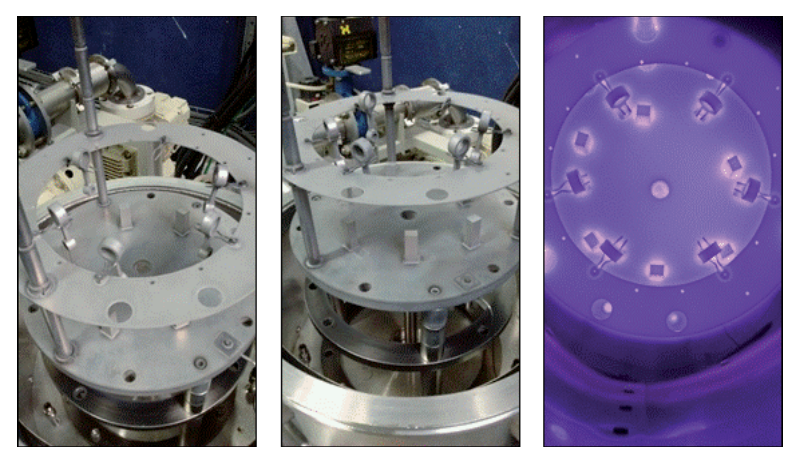

Figura 1: Vista do interior do reator a plasma usado na nitrocarbonetação das amostras.

Tabela 1: Composição química da amostra de liga ferrosa.

\begin{tabular}{|c|c|c|c|c|c|c|}
\hline \multirow{2}{*}{ DIN 100Cr6 } & \multicolumn{5}{|c|}{ Composição Química (\% em peso) } \\
\cline { 2 - 6 } & Fe & C & Cr & Mn & 0,15 a 0,35 \\
\hline Valor nominal (AISI) & Balanço & 0,98 a 1,10 & 1,30 a 1,60 & 0,25 a 0,45 & 0,25 \\
\hline Valor determinado & Balanço & 0,90 & 1,53 & 0,33 & . \\
\hline
\end{tabular}


Para a análise por microscopia eletrônica de varredura (MEV), utilizou-se o equipamento XL-30 FEG Philips com canhão de emissão por campo, equipado com um espectrômetro de energia dispersiva (EDS). As amostras embutidas foram previamente preparadas e recobertas com ouro e prata para permitir contato elétrico entre a amostra e o suporte da câmara. As imagens foram adquiridas empregando-se elétrons retroespalhados (BSE), que permitem a visualização de diferenças na composição química das amostras.

As análises de difratometria de raios $\mathrm{X}$ (DRX) foram realizadas utilizando-se o equipamento de raios X Rigaku modelo GeigerFlex, sob as seguintes condições: radiação $\mathrm{Cu}-\mathrm{K \alpha}$, com comprimento de onda $\lambda=1,54056 \AA$, inerente ao tubo de cobre; ângulo de varredura $(\theta-2 \theta)$ de $5^{\circ}$ a $90^{\circ}$; ângulo de avanço: $0,032^{\circ}$ s. Para a identificação das fases e a caracterização dos picos de difração, utilizou-se o programa Diffrac EVA Release 2001.

\section{RESULTADOS E DISCUSSÃO Análise por MEV/EDS}

A análise via MEV/EDS foi utilizada para determinação das espessuras, tanto da camada composta como da zona de difusão. O gráfico da Fig. 2 a seguir apresenta um comparativo da espessura da camada composta mais a espessura da camada de austenita transformada, encontrada para algumas amostras, onde é possível verificar que o aumento da temperatura de nitrocarbonetação aumenta a espessura da camada superficial formada.

O coeficiente de difusão dos elementos aumenta exponencialmente com o aumento da temperatura, portanto, para maiores temperaturas, maior a espessura da camada formada, justamente pela maior difusão presente. Para nitrocarbonetação em baixas temperaturas, a mobilidade de átomos de nitrogênio e carbono é baixa, dificultando o processo de difusão ${ }^{(6)}$.

Para a maioria das amostras, a morfologia da camada composta pode ser representada pela micrografia obtida por MEV da Fig. 3.

$\mathrm{Na}$ parte superior da camada composta, que contém a camada porosa, a fase predominante é a fase $\varepsilon$. Na parte inferior da camada composta, cujo aspecto da estrutura é de padrão colunar,

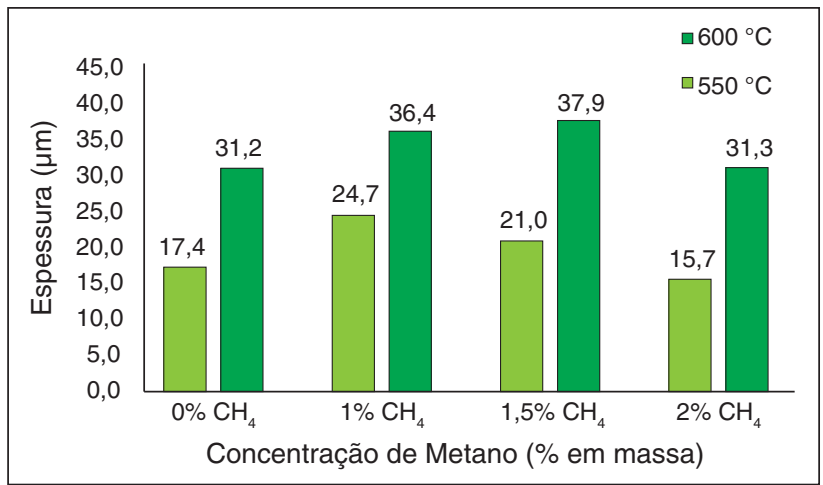

Figura 2: Comparativo da espessura da camada composta mais a camada de austenita transformada, em função da temperatura de nitrocarbonetação e concentração de $\mathrm{CH}_{4}$ na mistura gasosa. as fases predominantes são as fases $\varepsilon$ e $\gamma^{\prime}$, assim como investigado por Fattah e Mahboubi ${ }^{(7)}$, e Mittemeijer ${ }^{(8)}$.

A fase denominada de $\gamma^{\prime}$, considerada a fase mais estável do diagrama $\mathrm{Fe}-\mathrm{N}$, apresenta a estequiometria $\mathrm{Fe}_{4} \mathrm{~N}$, estrutura cristalina cúbica de face centrada (CFC), com o elemento $\mathrm{N}$ ocupando os interstícios octaédricos. Apresenta uma quantidade de nitrogênio na faixa de 19,4 - 20,0\% atômica e quantidade de carbono menor que $0,7 \%$ atômica ${ }^{(8)}$. A fase $\varepsilon$, que comparativamente é mais dura e resistente ao desgaste do que a fase $\gamma^{\prime}$, apresenta a fórmula não-estequiométrica $\mathrm{Fe}_{2-3} \mathrm{~N}$ e possui uma estrutura cristalina hexagonal compacta (HC) com o elemento $\mathrm{N}$ ocupando também os interstícios octaédricos. A quantidade de nitrogênio está na faixa de 15 - 33\% atômica e a quantidade de carbono menor que $8 \%$ atômica ${ }^{(8)}$.

Quanto à presença da camada porosa formada na superfície de todas as amostras, a Fig. 4 compara as espessuras para as diferentes temperaturas e concentrações de $\mathrm{CH}_{4}$ na mistura gasosa.

Como colocado por Li et. al. ${ }^{(9)}$, a composição da atmosfera apresenta uma forte influência na quantidade e formação dos poros da camada nitrocarbonetada. Uma atmosfera com alta concentração de nitrogênio resulta em maior quantidade de poros na camada composta, enquanto que uma menor concentração resultará em menor quantidade de poros na camada superficial.

Também, amostras nitrocarbonetadas na condição de maior temperatura, apresentaram uma camada de micro-porosidade

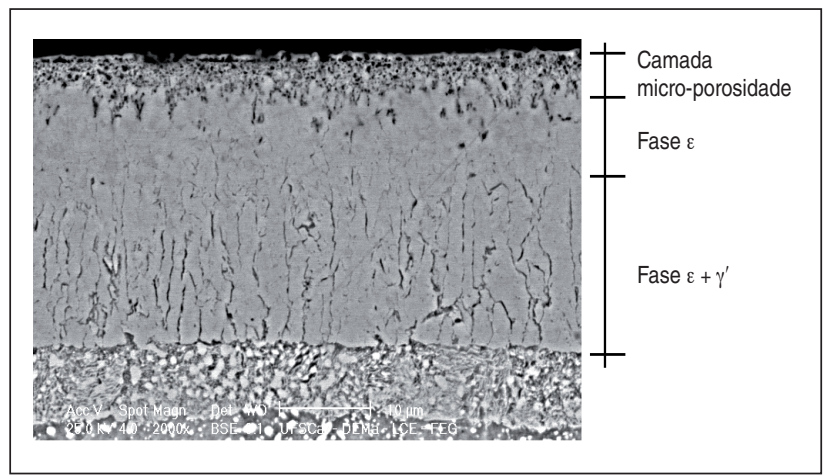

Figura 3: Detalhe da camada composta identificando as duas subcamadas presentes.

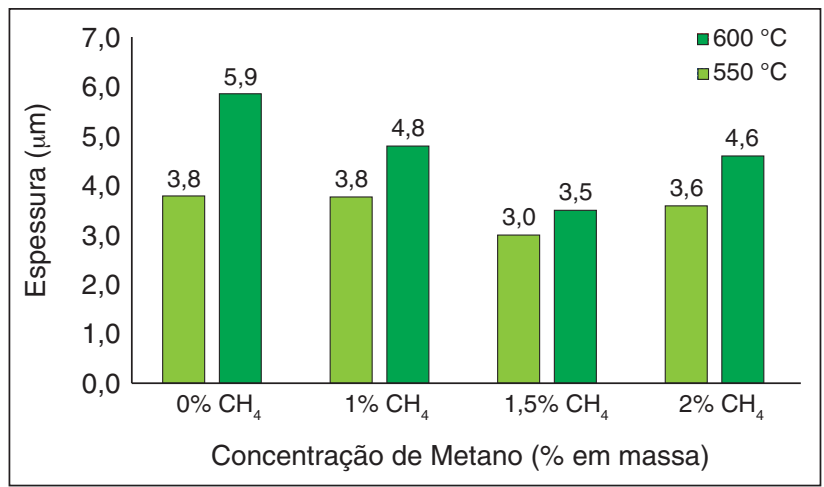

Figura 4: Espessura da camada de micro-porosidade, em função da temperatura e concentração de metano presente na mistura gasosa. 
maior que as amostras nitrocarbonetadas na condição de menor temperatura, para uma mesma concentração de $\mathrm{CH}_{4}$. Para que um poro seja desenvolvido na superfície da camada, é necessário que haja a absorção de moléculas de nitrogênio pela mesma, sendo que em baixas temperaturas a absorção é menor.

A presença da micro-porosidade não pode ser considerada um fenômeno necessariamente ruim. Como colocado por Hoffman et al. $^{(10)}$, a presença de uma zona porosa na camada superficial pode acelerar o desgaste inicial devido à baixa densidade presente e menor resistência à fadiga, porém segundo Karamis ${ }^{(1)}$ e Mittemeijer et al. ${ }^{(2)}$, a presença de porosidades funcionam como reservatórios de contenção de óleo, melhorando as condições de lubrificação.

Para as amostras tratadas a $600{ }^{\circ} \mathrm{C}$, é possível verificar a presença de uma camada de austenita transformada entre a camada composta e a zona de difusão, conforme ilustra a Fig. 5, assim como encontrada por Gao et al. ${ }^{(12)}$, Gontijo et al. ${ }^{(13)}$ e Fattah e Mahboubi ${ }^{(7)}$

Os valores das espessuras da camada de austenita transformada foram tabeladas e estão apresentadas na Fig. 6.

Pelos resultados, nota-se que o aumento na concentração de $\mathrm{CH}_{4}$ na mistura gasosa promove a diminuição da espessura da referida camada. A maior presença de carbono na atmosfera de tratamento, devido a maior quantidade de $\mathrm{CH}_{4}$, é responsável

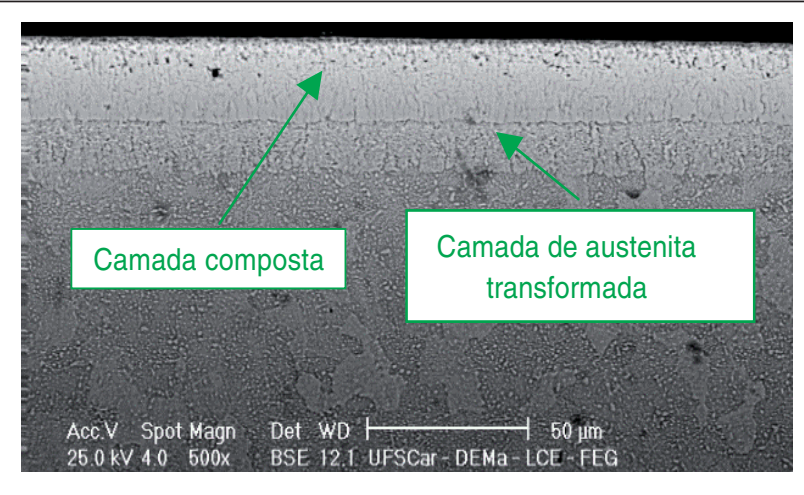

Figura 5: Amostra nitrocarbonetada a $600^{\circ} \mathrm{C}$ com $1 \% \mathrm{CH}_{4}$.

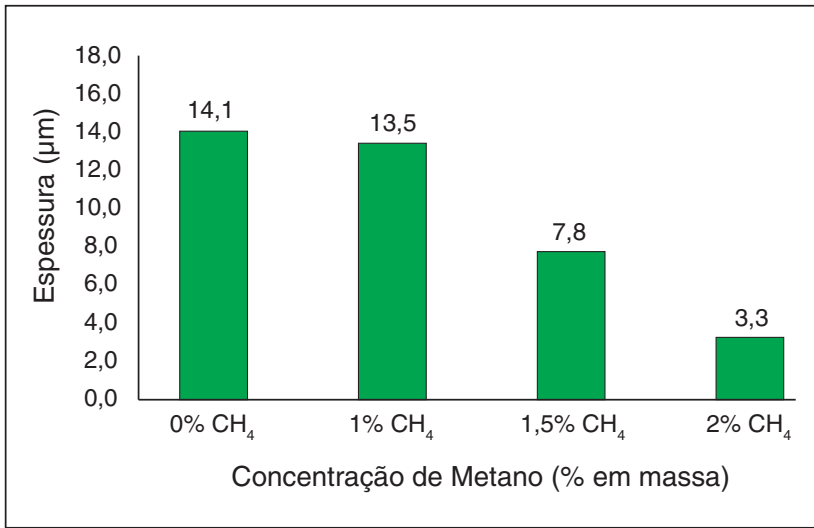

Figura 6: Comparativo da espessura da camada de austenita transformada para a temperatura de $600^{\circ} \mathrm{C}$, em função da concentração de $\mathrm{CH}_{4}$ na mistura. por aumentar a espessura da camada composta pela formação da combinação de fases $\varepsilon$ e $\gamma^{\prime}$, bem como facilitar o crescimento da zona de difusão, diminuindo como consequência a espessura da camada de austenita transformada.

Para determinação da espessura da zona de difusão, foi avaliado o perfil de composição química dos elementos carbono e nitrogênio através da análise por EDS (Figs. 7 e 8).

É possível verificar a presença de uma pequena quantidade de carbono e uma grande quantidade de nitrogênio na camada composta, justamente pela presença das fases $\varepsilon-\mathrm{Fe}_{3} \mathrm{~N}$ e $\gamma^{\prime}-\mathrm{Fe}_{4} \mathrm{~N}$ encontradas para todas as amostras nitrocarbonetadas em ambas as temperaturas. A baixa quantidade de carbono na camada composta é consequência da alta concentração de nitrogênio, devido a maior estabilidade termodinâmica dos nitretos em relação aos carbonitretos.

Na zona de difusão há uma inversão, ou seja, é possível verificar a presença de uma pequena quantidade de nitrogênio com uma grande quantidade de carbono, porém ambos os elementos diminuindo ao longo da linha de composição química, até alcançar a composição química do núcleo da amostra. Observa-se ainda nas Figs. 7 e 8 que a espessura da zona de difusão para amostras nitrocarbonetadas a $600{ }^{\circ} \mathrm{C}$ é bem maior que para amostras tratadas a $500{ }^{\circ} \mathrm{C}$, assim como foi observado para a camada composta.

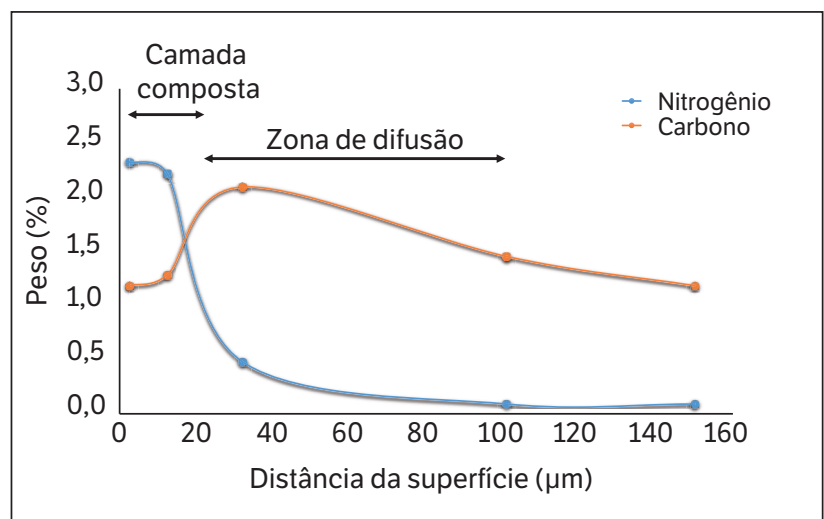

Figura 7: Perfil de composição química média para amostras tratadas na temperatura de $550^{\circ} \mathrm{C}$.

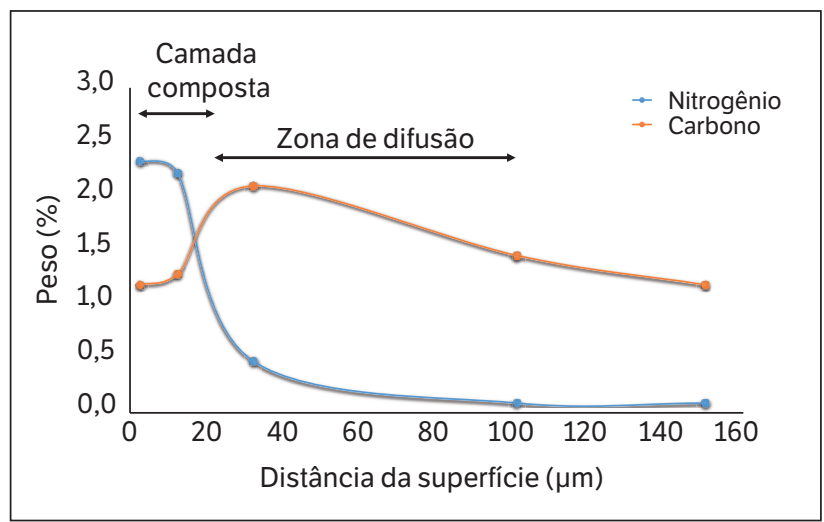

Figura 8: Perfil de composição química média para amostras tratadas na temperatura de $600{ }^{\circ} \mathrm{C}$. 


\section{Análise por DRX}

A Fig. 9 apresenta a análise de DRX para as amostras nitrocarbonetadas na temperatura de $550{ }^{\circ} \mathrm{C}$, para as quatro diferentes concentrações de $\mathrm{CH}_{4}$.

Fica evidente, ao analisar a Fig. 9, que a amostra nitrocarbonetada com $0 \%$ de $\mathrm{CH}_{4}$ apresentou uma grande quantidade da fase $\gamma^{\prime}$ e somente traços da fase $\varepsilon$. $O$ aumento da concentração de metano na mistura gasosa, com consequente aumento na quantidade de carbono na atmosfera de nitrocarbonetação promoveu o aumento na quantidade da fase $\varepsilon$ e uma diminuição da fase $\gamma$ '.

Conforme o estudo efetuado por Mittmemeijer ${ }^{(8)}$, existem diferentes caminhos de difusão quando se observa uma seção isotérmica do diagrama de fase $\mathrm{Fe}-\mathrm{C}-\mathrm{N}$ em uma determinada temperatura. Quanto maior a quantidade de carbono presente na atmosfera da mistura gasosa, menor a possibilidade de formação da fase $\gamma^{\prime}$, e consequentemente maior a possibilidade de formação de uma camada constituída apenas da fase $\varepsilon$.

Da mesma forma para amostras nitrocarbonetadas na temperatura de $600{ }^{\circ} \mathrm{C}$, a Fig. 10 também apresenta a análise de difratometria de raios $\mathrm{X}$ para as quatro diferentes concentrações de $\mathrm{CH}_{4}$ na mistura gasosa.

Assim como na análise das amostras nitrocarbonetadas a $550^{\circ} \mathrm{C}$, as amostras nitrocarbonetadas na temperatura de $600{ }^{\circ} \mathrm{C}$ também apresentam uma maior formação de fase $\varepsilon$ para atmosferas com

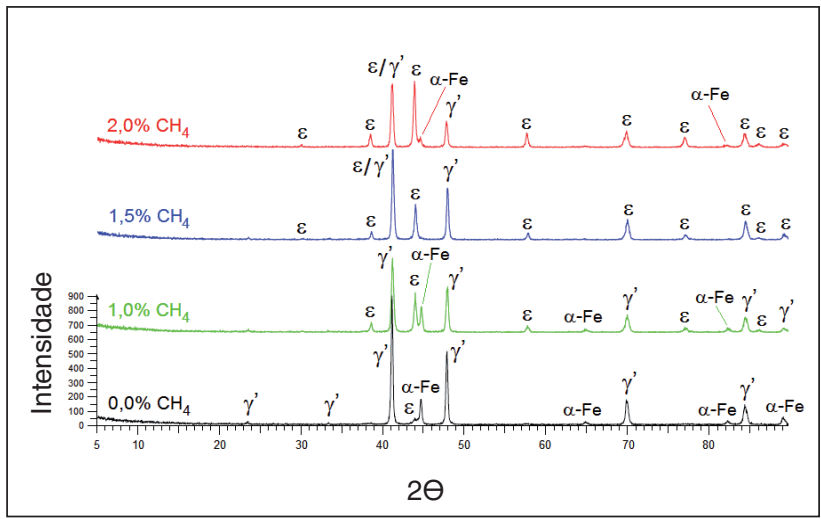

Figura 9: Difratogramas de raios $X$ para as amostras nitrocarbonetadas a $550{ }^{\circ} \mathrm{C}$.

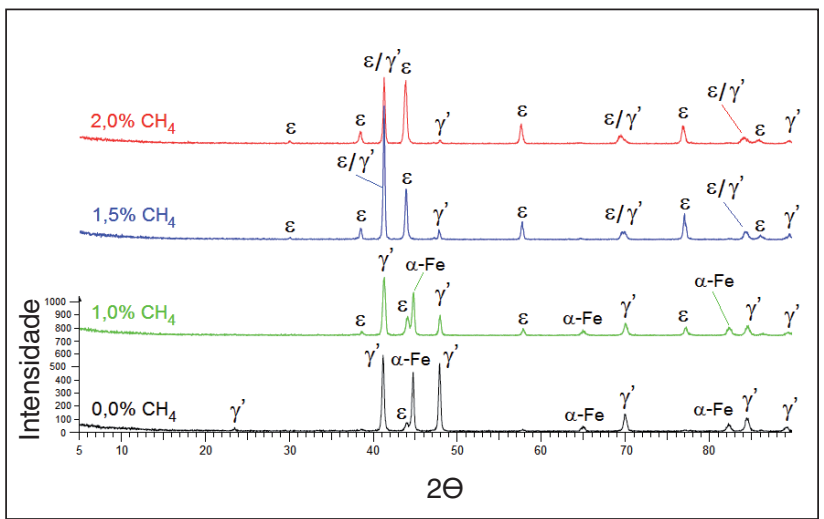

Figura 10: Difração de raio-X para as amostras nitrocarbonetadas a $600{ }^{\circ} \mathrm{C}$. maiores concentrações de $\mathrm{CH}_{4}$. A baixa concentração de metano na mistura gasosa favorece a formação de grande quantidade de fase $\gamma^{\prime}$ em suas microestruturas.

Comparando-se os raios-X das amostras nitrocarbonetadas a $550{ }^{\circ} \mathrm{C}$ e $600{ }^{\circ} \mathrm{C}$, nota-se que o aumento da temperatura promove uma maior formação de carbonitretos de fase $\gamma^{\prime}$ produzido juntamente com a fase $\varepsilon$. Isto se deve ao fato da maior decomposição da fase $\varepsilon$, de baixo teor de nitrogênio, em $\gamma^{\prime}, \varepsilon$ e $\mathrm{Fe}-\alpha$ durante o resfriamento lento da amostra, para amostras tratadas a $600{ }^{\circ} \mathrm{C}$.

Desta forma, comparando-se a nitrocarbonetação ferrítica (realizado abaixo de $590^{\circ} \mathrm{C}$ ) com a austenítica (realizado acima de $590^{\circ} \mathrm{C}$ ), pode-se afirmar que a nitrocarbonetação ferrítica é capaz de produzir uma camada composta com presença predominante de fase $\varepsilon$.

\section{CONCLUSÕES}

Os parâmetros envolvidos no tratamento de nitrocarbonetação, como temperatura e composição química da mistura gasosa, influenciam diretamente na morfologia da camada superficial formada. Conclui-se que as espessuras da camada composta e da zona de difusão aumentam com o aumento da temperatura de processo, devido ao coeficiente de difusão dos elementos carbono e nitrogênio aumentarem exponencialmente com o aumento da temperatura. A constituição da camada composta é bastante sensível à quantidade de carbono presente na atmosfera de tratamento, onde, de acordo com o diagrama de fases do sistema ternário Fe$\mathrm{C}-\mathrm{N}$, o carbono contribui para a formação da fase $\varepsilon$. Quanto maior a quantidade de carbono na mistura gasosa, maior a possibilidade de formação de uma camada constituída apenas pela fase $\varepsilon$.

Para amostras nitrocarbonetadas a $600^{\circ} \mathrm{C}$, notou-se a formação de uma camada de austenita transformada entre a camada composta e a zona de difusão, justamente porque o processo foi realizado acima da temperatura de transformação da austenita $\left(590{ }^{\circ} \mathrm{C}\right)$. Após o resfriamento lento dentro do reator, a camada formada transformou-se na fase $\gamma^{\prime}$, e sua espessura foi inversamente proporcional à concentração de $\mathrm{CH}_{4}$ presente na mistura gasosa.

Comparando-se os resultados da análise de DRX das amostras nitrocarbonetadas com $0 \% \mathrm{CH}_{4}$, com os resultados das amostras nitrocarbonetadas em uma mistura gasosa com diferentes quantidades de $\mathrm{CH}_{4}$, nota-se que a camada composta


basicamente da fase $\gamma^{\prime}$, com apenas traços da fase $\varepsilon$. Com a introdução de $\mathrm{CH}_{4}$ na mistura gasosa, há o surgimento de uma camada combinada de fases $\varepsilon$ e $\gamma$ ', sendo uma camada mais externa formada somente pela fase $\varepsilon$ e uma outra camada mais interna, em direção ao núcleo das amostras, formada por ambas as fases $\varepsilon$ e $\gamma^{\prime}$ com uma microestrutura de aspecto tipo colunar.

Uma camada de micro-porosidade foi formada para todas as amostras, sendo que para atmosferas com $0 \% \mathrm{CH}_{4}$, as camadas de micro-porosidades foram maiores, assim como para amostras 
nitrocarbonetadas nas maiores temperaturas. Altas concentrações de nitrogênio, bem como maiores temperaturas favorecem a absorção das moléculas de nitrogênio na camada superficial formada, favorecendo como consequência a produção da camada de micro-porosidade.

Conclui-se então que a escolha correta dos parâmetros temperatura e mistura gasosa da atmosfera de tratamento, é essencial para promover a melhor morfologia da camada superficial formada. A microestrutura e a morfologia da camada são responsáveis pelos resultados das propriedades tribológicas e mecânicas, e definirão os níveis de confiabilidade e os valores de vida útil dos componentes que utilizam do processo de nitrocarbonetação a plasma.

\section{AGRADECIMENTOS}

Os autores gostariam de agradecer Fernando Nawate da UFSCar/DEMa pela assistência nas medições do MEV/EDS.

Este trabalho teve apoio do $\mathrm{CNPq}$ (processo n ${ }^{\circ} 304555 / 2013-4$ ).

\section{REFERÊNCIAS}

1. JACK, K. H., Results of further X-ray structural investigations of the Iron-Carbon and Iron-Nitrogen system and of related interstitial alloys. Acta Crystallographica, v.3, n.5, p.392-394, 1950.

2. MITTEMEIJER, E. J.; SOMERS, M. A. J., Thermochemical surface engineering of steels. Cambridge, Woodhead Publishing Series in Metals and Surface Engineering, 2015
3. HOPPE, S., Fundamentals and applications of the combination of plasma nitrocarburizing and oxidizing. Surface and Coatings Technology, v.98, n.1-3, p.1199-1204, 1998.

4. MERLO, A. M., The contribution of surface engineering to the product performance in the automotive industry. Surface and Coatings Technology, v.174-175, p.21-26, 2003.

5. LAMPE, TH.; EISENBERG, S.; RODRIGUEZ CABEO, E., Plasma surface engineering in the automotive industry - trends and future prospective. Surface and Coatings Technology, v.174-175, p.1-7, 2003.

6. MAHBOUBI, F.; ABDOLVAHABI, K., The effect of temperature on plasma nitriding behaviour of DIN 1.6959 low alloy steel. Vacuum, v.81, n.3, p.239-243, 2006.

7. FATTAH, M.; MAHBOUBI, F., Microstructure characterization and corrosion properties of nitrocarburized AISI 4140 low alloy steel. Journal of materials engineering and performance, v.21, n.4, p.548-552, 2012

8. MITTEMEIJER, E. J., Steel Heat Treating Fundamentals and Processes. ASM Handbook A, v.4, 2013.

9. LI, S.; MANORY, R. R., Surface morphology and compound layer pores of plasma nitrocarburized low carbon steel. Metallurgical and Materials Transactions A, v.27, n.1, p.135-143, 1996

10. HOFFMANN, F. T.; MAYR, P., Nitriding and nitrocarburizing. ASM Handbook, v.18, p.878-883, 1992.

11. KARAMIS, M.B., Wear properties of steel plasma nitrided at high temperatures. Materials Science and Engineering: A, v.168, n.1, p.49-53, 1993

12. GAO, Y.; GUO, X. P.; WEI, R. . Rapid nitriding of pure iron by thermal plasma jet irradiation. Surface \& Coatings Technology, v.201, p.2829-2834, 2006

13. GONTIJO, L. C. et al., Characterization of plasma-nitrided iron by XRD, SEM and XPS. Surface \& Coatings Technology, v.183, p.10-17, 2004. 